\title{
Delirium in hemodialysis predicts mortality: a single-center, long-term observational study
}

This article was published in the following Dove Press journal:

Neuropsychiatric Disease and Treatment

\author{
Norio Yasui-Furukori ${ }^{1}$ \\ Natsumi Tarakita' \\ Waka Uematsu² \\ Hisao Saito ${ }^{2}$ \\ Kazuhiko Nakamura' \\ Chikara Ohyama ${ }^{3}$ \\ Norio Sugawara ${ }^{4}$ \\ 'Department of Neuropsychiatry, \\ Graduate School of Medicine, \\ Hirosaki University, Hirosaki, \\ ${ }^{2}$ Department of Urology, Oyokyo \\ Kidney Research Institute, Hirosaki, \\ ${ }^{3}$ Department of Urology, Graduate \\ School of Medicine, Hirosaki \\ University, Hirosaki, ${ }^{4}$ Department of \\ Clinical Epidemiology, Translational \\ Medical Center, National Center of \\ Neurology and Psychiatry, Kodaira, \\ Tokyo, Japan
}

\footnotetext{
Correspondence: Norio Yasui-Furukori Department of Neuropsychiatry, Hirosaki University, School of Medicine, Hirosaki 036-8562, Japan

Tel +81172395066

Fax +8I 172395067

Email yasufuru@hirosaki-u.ac.jp
}

\begin{abstract}
Objectives: Delirium signifies underlying brain dysfunction; however, its clinical significance in hemodialysis remains unclear. In this study, we sought to determine whether the occurrence of delirium during hemodialysis was associated with higher mortality.

Patients and methods: This was a retrospective, 10-year cohort study. This study was performed at the urology department located within a hospital in Oyokyo, Hirosaki. We analyzed 338 of 751 patients who underwent hemodialysis. Psychiatrists diagnosed patients with delirium according to the corresponding DSM-IV-TR criteria. Cox proportional hazard regression, which was adjusted for patient age at the time of hemodialysis initiation, sex, and the presence of diabetes mellitus, was performed. Hazard ratios (HRs) and their 95\% CIs were also reported.

Results: In total, 286 patients without psychiatric diseases and 52 patients with delirium were evaluated. Eighty percent of patients with delirium died within 1 year of hemodialysis initiation, while only $22 \%$ of patients without delirium died within the same time period $(P<0.01)$. Kaplan-Meier plots demonstrated the existence of associations between delirium and all-cause mortality (global log-rank $P<0.001$ ), cardiovascular disease-related mortality (global log-rank $P<0.001$ ), and infection-related mortality (global log-rank $P<0.001$ ). Moreover, Cox proportional hazard regression showed that delirium was associated with all-cause mortality $(\mathrm{HR}=1.96$, 95\% CI: 1.32-2.90), cardiovascular disease-related mortality (HR=2.65, 95\% CI: 1.31-5.35), and infection-related mortality ( $\mathrm{HR}=3.30,95 \% \mathrm{CI}$ : $1.34-8.10)$.

Conclusion: Delirium is an independent predictor of death in patients undergoing hemodialysis.
\end{abstract}

Keywords: delirium, hemodialysis, mortality, observational study, disturbance of consciousness

\section{Introduction}

Delirium is a neurobehavioral syndrome characterized by fluctuating or abnormal mental status and inattention as well as an altered level of consciousness or disorganized thinking. ${ }^{1}$ Delirium is a common complication affecting patients in the intensive care unit ${ }^{2}$ and is associated with higher mortality, prolonged hospitalization, and long-term cognitive impairment. ${ }^{3,4}$ Clinicians have attributed the disease to several phenomena, including neurotransmitter imbalances, sleep-wake cycle disruption, metabolic abnormalities, and stress responses $;{ }^{5}$ however, the pathogenesis of delirium remains unclear.

Chronic kidney disease affects $\sim 10 \%-15 \%$ of adults globally and increases the risk of death by $\sim 2-4$-fold in affected patients, compared with that in unaffected patients. This increase has been attributed mostly to increased cardiovascular diseaserelated mortality. ${ }^{6-9}$ Patients with stage 5 chronic kidney disease who are receiving dialysis treatment display a particularly heavy symptom burden. Specifically, these patients develop fatigue, anxiety and depression, sleep disturbances, and itching. ${ }^{10}$ 
Delirium is commonly observed in patients undergoing dialysis. It has been attributed to electrolyte imbalances that occur after dialysis, which cause a phenomenon known as dialysis disequilibrium syndrome (DDS), or because of medical or surgical complications. ${ }^{11,12}$ Delirium may also be caused by uremia, anemia, and hyperparathyroidism.

Few studies, however, have explored whether the presence of delirium is associated with mortality in patients undergoing hemodialysis over a long-term observation period. Therefore, we assessed patients with known psychiatric diagnoses of delirium and examined the association between delirium and mortality in a cohort of patients undergoing maintenance hemodialysis over 10 years of observation.

\section{Patients and methods}

\section{Study sample and group assignment}

The study protocol was approved by the Ethics Committee of Hirosaki University on Human Research. Informed consent was obtained from all patients. None of the patients refused to participate in the retrospective study. Between 2006 and 2015, 751 consecutive patients undergoing hemodialysis were approached and asked for their consent to collect their clinical data for research purposes, retrospectively. Of these 751 eligible patients, 492 received an early psychiatric diagnostic assessment, 346 did not exhibit symptoms of psychiatric disease, and 146 displayed symptoms of psychiatric disease (Figure 1). Among 146 patients, 39 who presented with delirium upon initiation, as determined by trained psychiatrists according to the corresponding DSM-IV criteria, were eligible for the study. Moreover, the remaining 107 patients displaying symptoms of psychiatric disease, whose diagnoses included dementia $(n=39)$, insomnia $(n=25)$, depression $(n=19)$, and other psychiatric diseases ( $n=24$; epilepsy, schizophrenia, neuropathic pain, adjustment disorder, and panic disorder), were excluded from the analyses. After hemodialysis initiation, 60 of the abovementioned 346 patients without a previous history of psychiatric symptoms or disease exhibited symptoms of psychiatric disease. Thirteen of these patients received diagnoses of delirium, according to the criteria specified by the DSM-IV and, thus, were included in the study. The remaining 47 patients, whose diagnoses included dementia $(n=20)$, insomnia $(n=10)$, depression $(n=8)$, and other psychiatric diseases $(n=9$; epilepsy, schizophrenia, neuropathic pain, and adjustment disorder), were excluded from the analyses. Thus, 286 healthy patients and 52 patients with delirium were ultimately included in this study.

We obtained demographic data (age, sex) and information pertaining to clinical diagnoses by reviewing the Oyokyo Hospital database and patient medical records (Table 1). Data regarding overall and specific mortality rates were obtained from the clinical database and patient death certificates. Cardiovascular

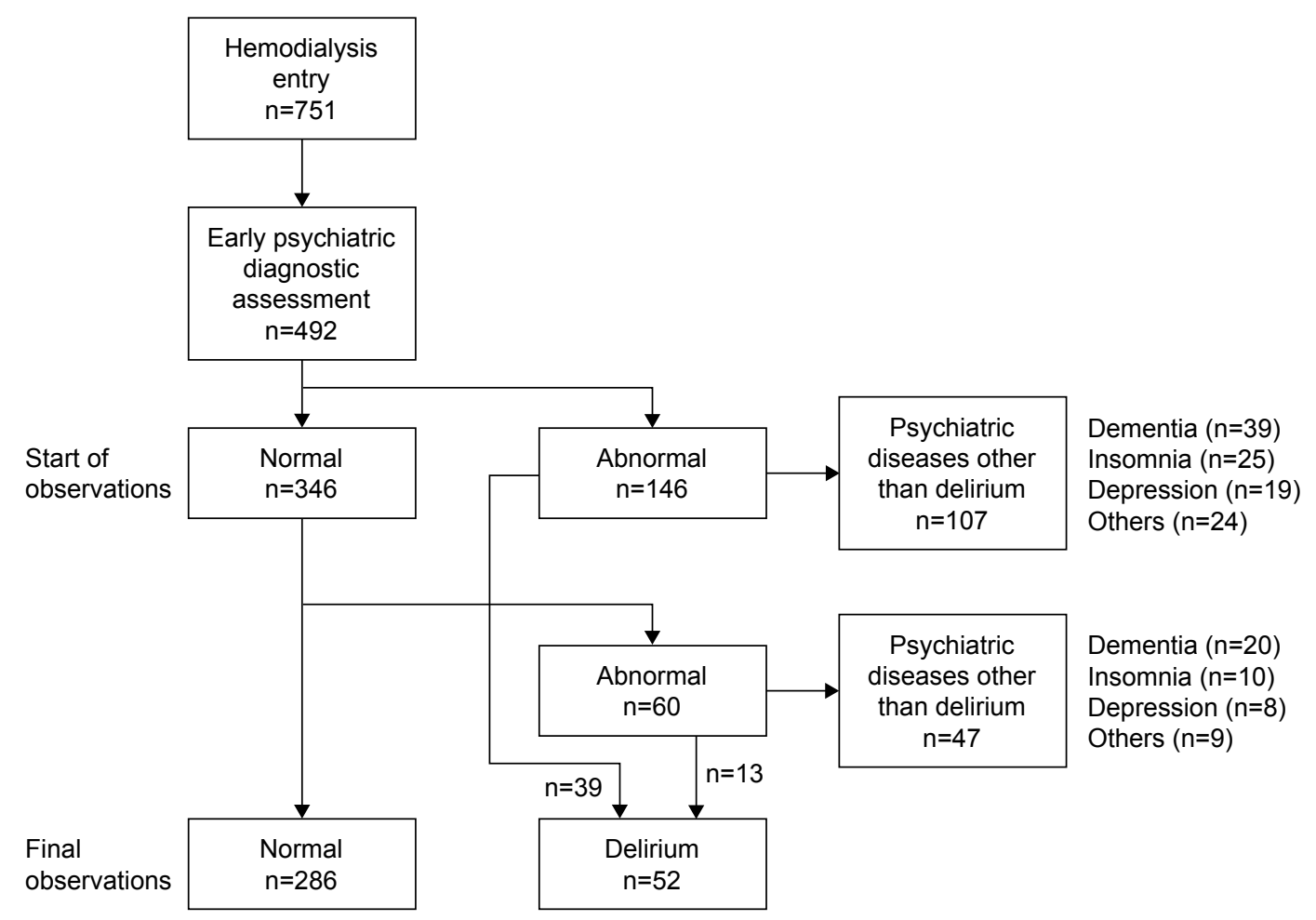

Figure I Flow chart of patient inclusion in the study. 
Table I Patient characteristics

\begin{tabular}{llll}
\hline & $\begin{array}{l}\text { Control, } \\
\mathbf{n}=\mathbf{2 8 6}(\%)\end{array}$ & $\begin{array}{l}\text { Delirium, } \\
\mathbf{n}=\mathbf{5 2} \text { (\%) }\end{array}$ & $\begin{array}{l}\text { Pignificance, } \\
\text {-value }\end{array}$ \\
\hline Age at entry (years) $^{\mathrm{a}}$ & $65.8 \pm 12.7$ & $75.4 \pm 8.1$ & $<0.00 \mathrm{I}$ \\
Male sex & $\mathrm{I} 88(66)$ & $29(56)$ & 0.168 \\
Duration of hemodialysis (days) & $825 \pm 806$ & $884 \pm 814$ & 0.623 \\
Primary indication of hemodialysis & & \\
Diabetes & $174(60.8)$ & $27(51.9)$ & \\
Hypertension & $23(8.0)$ & $5(9.6)$ & \\
Glomerulonephritis & $20(7.0)$ & $\mathrm{I}(1.9)$ & 0.206 \\
Others & $25(8.7)$ & $5(9.6)$ & \\
Unknown & $44(15.4)$ & $14(26.9)$ & \\
Primary cause of death & $\mathrm{n}=8 \mathrm{I}$ & $\mathrm{n}=39$ & \\
Cardiovascular disease & $21(25.9)$ & $14(36.8)$ & \\
Infection & $10(12.3)$ & $10(26.3)$ & \\
Cancer & $13(16.0)$ & $1(2.6)$ & $<0.00 \mathrm{I}$ \\
Others & $19(23.5)$ & $13(34.2)$ & \\
Unknown & $18(22.2)$ & $0(0.0)$ & \\
\hline Note & & &
\end{tabular}

Note: aData shown as mean \pm SD

disease-related causes of death included sudden death or acute myocardial infarction-related death; pericarditis; atherosclerotic heart disease; cardiomyopathy; cardiac arrhythmia; cardiac arrest; valvular heart disease; pulmonary edema; congestive cardiac failure; cerebrovascular accidents, including intracranial hemorrhage; and ischemia-induced brain injury-related phenomena, including anoxic encephalopathy. The primary outcomes of the study, which were defined a priori, were allcause mortality and death due to cardiovascular disease-related causes and infection-related mortality. Patients were followed until their death. Survival time was defined as the period between each patient's initial enrollment (starting hemodialysis) in the study and his or her death or December 31, 2015.

\section{Statistical analysis}

The descriptive characteristics of the study population were reported as proportions in the case of categorical and binary variables, means and SDs in the case of continuous normally distributed variables, and medians with interquartile ranges in the case of skewed variables. Differences between categorical variables were assessed using chi-squared tests. Mortality rates were calculated by dividing the number of deaths in each group by the total accrued person-time in each group. Kaplan-Meier plots for time to death were constructed for the healthy patient and delirium patient groups, and the log-rank test was used to determine the difference in survival between the healthy patient and delirium patient groups. Cox proportional hazards regression models were used to assess the association between the presence of delirium and all-cause mortality, cardiovascular-related mortality, and infectionrelated mortality, and nested models were used to perform adjustments for demographics (age, sex, and the presence of diabetes). A $P$-value $<0.05$ was regarded as statistically significant. The covariates served as time-dependent variables. All analyses were performed using SPSS, version 24.

\section{Results}

Two hundred eighty-six patients without psychiatric disease and 52 patients with delirium were analyzed. The group of patients with delirium was significantly older than the group of patients without delirium $(P<0.001$; Table 1$)$. The average duration of hemodialysis was not different between the patients with delirium and those without delirium (Table 1). Our results showed no sex distribution between the delirium group and the healthy group (Table 1). No difference in the frequency of underlying disease was found between the healthy group of patients and the group of patients with delirium; however, there was a significant difference between the two groups with respect to the incidence of specific causes of death $(P<0.01$; Table 1$)$. There was no difference in characteristic data between the early detection group $(n=39)$ and the late detection group $(n=13$; data not shown). Delirium was treated with antipsychotics such as haloperidol $(n=25)$, risperidone $(n=15)$, quetiapine $(\mathrm{n}=10)$, levomepromazine $(\mathrm{n}=10)$, perospirone $(\mathrm{n}=5)$, and blonanserin $(\mathrm{n}=4)$ and antidepressants such as trazodone $(n=3)$ and mianserin $(n=1)$. The analysis showed that $80 \%$ of patients with delirium died within 1 year of dialysis initiation, while only $22 \%$ of patients without delirium died during the same period $(P<0.01)$.

Figure 2 displays the unadjusted all-cause survival curves for the patients with and without delirium. The Kaplan-Meier plots demonstrated the existence of an association between

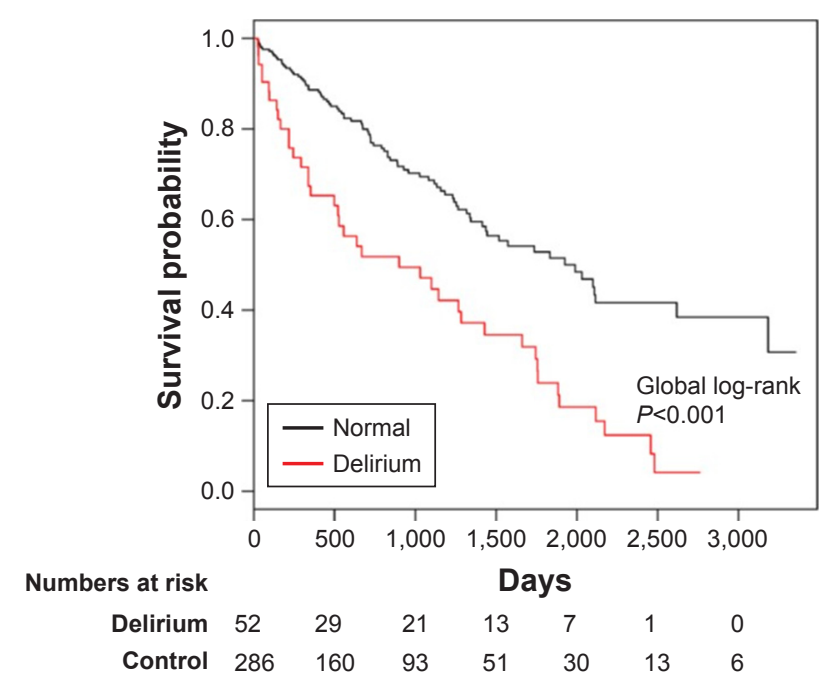

Figure 2 Unadjusted all-cause survival curves for patients with and without delirium. Note: The black line represents the survival curve for patients without delirium, and the red line represents the survival curve for patients with delirium. 
Table 2 Cox regression model for all-cause mortality, cardiovascular-related mortality, and infection-related mortality

\begin{tabular}{|c|c|c|c|c|c|c|}
\hline & \multicolumn{2}{|c|}{ All-cause mortality } & \multicolumn{2}{|c|}{ Cardiovascular-related mortality } & \multicolumn{2}{|c|}{ Infection-related mortality } \\
\hline & Crude model & Adjusted model & Crude model & Adjusted model & Crude model & Adjusted model \\
\hline Presence of delirium & $2.42(1.66-3.53)^{*}$ & $1.96(1.32-2.90) * *$ & $3.36(I .7 I-6.64)^{* * *}$ & $2.65(1.3 \mathrm{I}-5.35)^{* *}$ & $5.14(2.13-12.37)^{* * *}$ & $3.30(1.34-8.10)^{* *}$ \\
\hline Age & & $1.05(1.03-1.07)^{* * *}$ & & $1.09(1.05-1.14)^{* * *}$ & & I.II (I.05-I.I7) *** $^{* *}$ \\
\hline Male sex & & $1.86(1.26-2.73)^{* *}$ & & $1.77(0.87-3.62)$ & & $1.55(0.62-3.91)$ \\
\hline $\begin{array}{l}\text { Diabetes (as an indication } \\
\text { of hemodialysis) }\end{array}$ & & $1.24(0.84-1.83)$ & & $1.75(0.84-3.66)$ & & $1.15(0.45-2.96)$ \\
\hline
\end{tabular}

Notes: All variables are hazard ratio and $95 \% \mathrm{Cl}$. $* \mathrm{P}<0.05, * * \mathrm{P}<0.0 \mathrm{I}, * * * \mathrm{P}<0.00 \mathrm{I}$.

the presence of delirium and all-cause mortality (global log-rank $P<0.001$ ), and Cox proportional hazard regression showed that all-cause mortality was associated with the presence of delirium (hazard ratio $[\mathrm{HR}]=1.96,95 \% \mathrm{CI}$ : 1.32-2.90), age (HR=1.05, 95\% CI: $1.03-1.07)$, and male sex $(\mathrm{HR}=1.86,95 \% \mathrm{CI}: 1.26-2.73)$, but not the presence of diabetes (HR=1.24, 95\% CI: $0.84-1.83$ ), as shown in Table 2. Figure 3 displays the unadjusted cardiovascular disease-related survival curves for the patients with and without delirium. The Kaplan-Meier plots demonstrated the existence of an association between the presence of delirium and cardiovascular disease-related mortality (global log-rank $P<0.001$ ). Cox proportional hazard regression showed that cardiovascular disease-related mortality was associated with the presence of delirium ( $\mathrm{HR}=2.65,95 \%$ CI: $1.31-5.35)$ and age (HR=1.09, 95\% CI: $1.05-1.14)$, but not sex (HR=1.77, 95\% CI: $0.87-3.62)$ or the presence of diabetes (HR $=1.75,95 \% \mathrm{CI}$ : $0.84-3.66$ ), as shown in Table 2. Figure 4 displays the unadjusted infection curves for the patients with and without delirium. The Kaplan-Meier

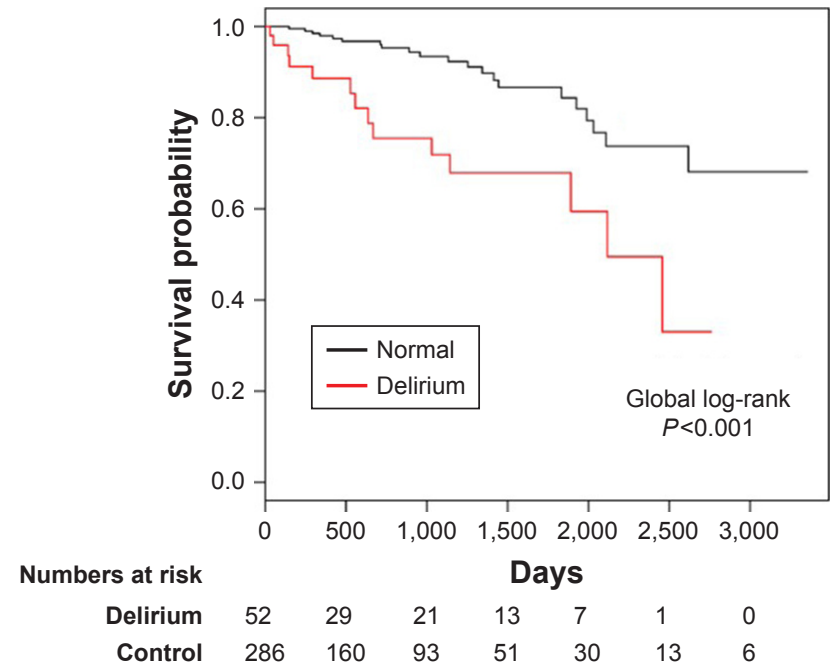

Figure 3 Unadjusted cardiovascular disease-related survival curves for patients with and without delirium.

Note: The black line represents the survival curve for patients without delirium, and the red line represents the survival curve for patients with delirium. plots demonstrated the existence of an association between the presence of delirium and infection-related mortality (global log-rank $P<0.001$ ). Cox proportional hazard regression showed that infection-related mortality was associated with the presence of delirium (HR=3.30, 95\% CI: 1.34-8.10) and age (HR=1.11, 95\% CI: $1.05-1.17)$, but not sex $(\mathrm{HR}=1.55,95 \% \mathrm{CI}: 0.62-3.91)$ or the presence of diabetes (HR=1.15, 95\% CI: $0.45-2.96$ ), as shown in Table 2 .

\section{Discussion}

There are limited data regarding the relationship between delirium and mortality in patients undergoing hemodialysis. In this longitudinal study, which examined the relationship between delirium and mortality in patients undergoing maintenance dialysis, the presence of delirium was found to be associated with an increased risk of mortality. The relationships between delirium and all-cause and cardiovascular- and infection-related mortality were attenuated by adjustments for demographics, but remained significant after adjustments for age, sex, and comorbidity of diabetes.

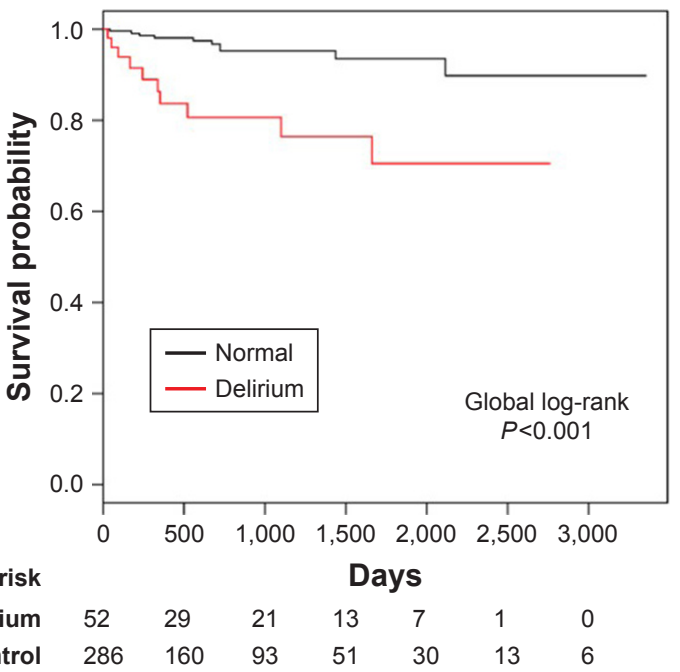

Figure 4 Unadjusted infection-related survival curves for patients with and without delirium.

Note: The black line represents the survival curve for patients without delirium, and the red line represents the survival curve for patients with delirium. 
It is difficult to distinguish between DDS, which is an adverse effect of acute hemodialysis in advanced uremic patients and is caused by brain edema, ${ }^{12}$ and delirium because some symptoms are very similar. DDS is reversible, ${ }^{12}$ whereas most patients died even in early detection delirium groups in the study. Therefore, it is unlikely that many subjects with DDS were included in the study, although we did not confirm DDS using brain imaging.

In this study, we noted significant associations between delirium and cardiovascular- and infection-related mortality. Medications such as antipsychotics may be useful in ameliorating delirium. ${ }^{13}$ However, most antipsychotics have an unfavorable cardiovascular side effect profile, and sudden cardiac death may occur even in patients with no cardiovascular disease risk factors. Currently, most clinicians are focusing on antipsychotic-induced corrected QT prolongation because this phenomenon may increase the risk of Torsades de Pointes and, eventually, sudden cardiac death. Other cardiovascular diseases attributed to antipsychotic use include the Bragada syndrome phenotype, myocardial infarction, and myocarditis. ${ }^{14}$ In addition, antipsychotic use was associated with a higher risk of pneumonia in an Alzheimer's disease cohort and a somewhat higher risk of pneumonia in a nonAlzheimer's disease cohort. ${ }^{15}$ Therefore, using antipsychotics as a standard treatment for delirium may lead to cardiovascular- and infection-related mortality.

Two studies explored the association between dementia, which was diagnosed via reviews of medical charts or billing codes, and mortality. Both studies noted that dementia was an independent risk factor for mortality. ${ }^{16,17}$ Based on these findings, we excluded patients with dementia from this study. Patients undergoing hemodialysis exhibit impairment in multiple cognitive function domains; however, executive function, which is critical for planning and performing tasks, appears to be affected more than other domains, such as memory. ${ }^{18}$ Our previous study showed that undergoing hemodialysis was a significant independent risk factor for lower Mini Mental State Examination scores. ${ }^{19}$ The causes of cognitive impairment in patients undergoing dialysis appear to be multifactorial; ${ }^{20}$ however, cerebrovascular disease is likely an important cause of such impairment ${ }^{21}$ because it often affects brain structures related to executive function. ${ }^{22}$

In this study, male sex was associated with all-cause and cardiovascular disease-related mortality. Three previous studies ${ }^{18,23,24}$ analyzed the risk of mortality in adult male versus female patients undergoing hemodialysis. Carrero et al used the large dataset of the European Renal Association European Dialysis and Transplant Association registry and found that younger women have a higher risk of dying from noncardiovascular causes than younger men. ${ }^{23}$ This finding could not be confirmed in this study involving patients receiving maintenance hemodialysis because the incidence of specific causes of death among patients aged 18-44 years did not differ significantly between men and women.

We excluded depression from the analysis because we aimed to exclude any non-delirium factors influencing mortality from the study; however, the prevalence of depression in patients undergoing dialysis appears to range from $15 \%$ to $30 \%$, depending on the methodology used to diagnose depression and demographics of the populations under study. ${ }^{25,26}$ Several previous studies whose cumulative population exceeded that of existing studies showed that an association exists between depression and cardiovascular mortality in adults with kidney failure. ${ }^{27-29}$

The findings of this study should be interpreted with caution for several reasons. First, the single-center longitudinal design of the study did not allow us to make causal assumptions regarding the relationship between delirium and mortality. Future multicenter longitudinal studies are needed to investigate these associations. Second, several potential confounding factors, such as physical activity levels, socioeconomic status, lifestyle, electrolyte abnormalities, body mass index, blood pressure, hemoglobin, albumin levels, and renal function, were not assessed in our study. Future studies adjusting for these confounders are needed. Third, early psychiatric diagnostic assessments were not performed in all patients because some of the patients were too ill to undergo a psychiatric examination. Therefore, some of the patients who were excluded from the study may have had more severe disease than those who were ultimately included in the study. Finally, as our sample size was relatively small, we could not completely rule out beta error as the reason for not detecting associations, such as the presence of diabetes, between the presence of delirium and mortality.

\section{Conclusion}

The presence of delirium has a significant and negative impact on mortality among patients undergoing hemodialysis. Physicians must promote early screening and the best preventive practices to ensure that affected patients receive optimal treatment.

\section{Acknowledgments}

The authors would like to thank Dr Yasushi Sato and Tetsu Tomita of Hirosaki University Hospital for their skillful contributions to this study. Norio Yasui-Furukori has 
received grant/research support or honoraria from and has been a lecturer for Dainippon Pharmaceutical and Mochida Pharmaceutical.

\section{Disclosure}

The authors report no conflicts of interest in this work.

\section{References}

1. Ely EW, Gautam S, Margolin R, et al. The impact of delirium in the intensive care unit on hospital length of stay. Intensive Care Med. 2001; 27(12):1892-1900.

2. Milbrandt EB, Deppen S, Harrison PL, et al. Costs associated with delirium in mechanically ventilated patients. Crit Care Med. 2004; 32(4):955-962.

3. Girard TD, Jackson JC, Pandharipande PP, et al. Delirium as a predictor of long-term cognitive impairment in survivors of critical illness. Crit Care Med. 2010;38(7):1513-1520.

4. Ely EW, Shintani A, Truman B, et al. Delirium as a predictor of mortality in mechanically ventilated patients in the intensive care unit. JAMA. 2004;291(14):1753-1762.

5. Simone MJ, Tan ZS. The role of inflammation in the pathogenesis of delirium and dementia in older adults: a review. CNS Neurosci Ther. 2011;17(5):506-513.

6. Zhang L, Wang F, Wang L, et al. Prevalence of chronic kidney disease in China: a cross-sectional survey. Lancet. 2012;379(9818):815-822.

7. Chadban SJ, Briganti EM, Kerr PG, et al. Prevalence of kidney damage in Australian adults: the AusDiab kidney study. J Am Soc Nephrol. 2003;147(Suppl 2):S131-S138

8. Coresh J, Selvin E, Stevens LA, et al. Prevalence of chronic kidney disease in the United States. JAMA. 2007;298(17):2038-2047.

9. Singh NP, Ingle GK, Saini VK, et al. Prevalence of low glomerular filtration rate, proteinuria and associated risk factors in North India using Cockcroft- Gault and Modification of Diet in Renal Disease equation: an observational, cross-sectional study. BMC Nephrol. 2009;10:4.

10. Murtagh FE, Addington-Hall J, Higginson IJ. The prevalence of symptoms in end-stage renal disease: a systematic review. Adv Chronic Kidney Dis. 2007;14(1):82-99.

11. Levy NB. Psychopharmacology in patients with renal failure. Int $J$ Psychiatr Med. 1990;20(4):325-334.

12. Hinchey J, Chaves C, Appignani B, et al. A reversible posterior leukoencephalopathy syndrome. N Engl J Med. 1996;334(8):494-500.

13. De Sousa A. Psychiatric issues in renal failure and dialysis. Indian $J$ Nephrol. 2008;18(2):47-50.

14. Polcwiartek C, Kragholm K, Schjerning O, Graff C, Nielsen J. Cardiovascular safety of antipsychotics: a clinical overview. Expert Opin Drug Saf. 2016;15(5):679-688.
15. Tolppanen AM, Koponen M, Tanskanen A, et al. Antipsychotic use and risk of hospitalization or death due to pneumonia in persons with and those without alzheimer disease. Chest. 2016;150(6):1233-1241.

16. Rakowski DA, Caillard S, Agodoa LY, Abbott KC. Dementia as a predictor of mortality in dialysis patients. Clin J Am Soc Nephrol. 2006;1(5):1000-1005.

17. Kurella M, Mapes DL, Port FK, Chertow GM. Correlates and outcomes of dementia among dialysis patients: the Dialysis Outcomes and Practice Patterns Study. Nephrol Dial Transplant. 2006;21(9):2543-2548.

18. Villar E, Remontet L, Labeeuw M, Ecochard R. Effect of age, gender, and diabetes on excess death in end-stage renal failure. $J \mathrm{Am} S o c$ Nephrol. 2007;18(7):2125-2134.

19. Odagiri G, Sugawara N, Kikuchi A, et al. Cognitive function among hemodialysis patients in Japan. Ann Gen Psychiatry. 2011;10:20.

20. Weiner DE, Scott TM, Giang LM, et al. Cardiovascular disease and cognitive function in maintenance hemodialysis patients. Am J Kidney Dis. 2011;58(5):773-781.

21. Sarnak MJ, Tighiouart H, Scott TM, et al. Frequency of and risk factors for poor cognitive performance in hemodialysis patients. Neurology. 2013;80(5):471-480.

22. Pereira AA, Weiner DE, Scott T C, et al. Subcortical cognitive impairment in dialysis patients. Hemodial Int. 2007;11(3):309-314.

23. Carrero JJ, de Jager DJ, Verduijn M, et al. Cardiovascular and noncardiovascular mortality among men and women starting dialysis. Clin J Am Soc Nephrol. 2011;6(7):1722-1730.

24. Carrero JJ, de Mutsert R, Axelsson J, et al. Sex differences in the impact of diabetes on mortality in chronic dialysis patients. Nephrol Dial Transplant. 2011;26(1):270-276.

25. Riezebos RK, Nauta KJ, Honig A, Dekker FW, Siegert CE. The association of depressive symptoms with survival in a Dutch cohort of patients with end-stage renal disease. Nephrol Dial Transplant. 2010; 25(1):231-236.

26. Tomita T, Yasui-Furukori N, Sugawara N, et al. Prevalence of major depressive disorder among hemodialysis patients compared with healthy people in Japan using the Structured Clinical Interview for DSM-IV. Neuropsychiatr Dis Treat. 2016;12:2503-2508.

27. Palmer SC, Vecchio M, Craig JC, et al. Association between depression and death in people with CKD: a meta-analysis of cohort studies. Am J Kidney Dis. 2013;62(3):493-505.

28. Boulware LE, Liu Y, Fink NE, et al. Temporal relation among depression symptoms, cardiovascular disease events, and mortality in end-stage renal disease: contribution of reverse causality. Clin J Am Soc Nephrol. 2006;1(3):496-504.

29. Weisbord SD. Patient-centreed dialysis care: depression, pain, and quality of life. Semin Dial. 2016;29(2):158-164.
Neuropsychiatric Disease and Treatment

\section{Publish your work in this journal}

Neuropsychiatric Disease and Treatment is an international, peerreviewed journal of clinical therapeutics and pharmacology focusing on concise rapid reporting of clinical or pre-clinical studies on a range of neuropsychiatric and neurological disorders. This journal is indexed on PubMed Central, the 'PsycINFO' database and CAS,

\section{Dovepress}

and is the official journal of The International Neuropsychiatric Association (INA). The manuscript management system is completely online and includes a very quick and fair peer-review system, which is all easy to use. Visit http://www.dovepress.com/testimonials.php to read real quotes from published authors. 